\title{
NEURAL NETWORK BASED AUTOMATIC SEA ICE CLASSIFICATION FOR CL-POL RISAT-1 IMAGERY
}

\author{
Rudolf Ressel; Suman Singha; Susanne Lehner \\ Maritime Safety and Security Lab \\ Remote Sensing Technology Institute, German Aerospace Center (DLR) \\ Heinrich Focke Str. 4, 28199, Bremen, Germany \\ Rudolf.Ressel@dlr.de; Suman.Singha@dlr.de; Susanne.Lehner@dlr.de
}

\begin{abstract}
SAR Polarimetry has become a valuable tool in spaceborne SAR based sea ice analysis. The two major objectives in SAR based remote sensing of sea ice is on the one hand to have a large coverage of the imaged ground area, and on the other hand to obtain a radar response that carries as much information as possible. Whereas single-polarimetric acquisitions of existing sensors offer a wide coverage on the ground, dual polarimetric, or even better fully polarimetric data offer a higher information content which allows for a more reliable automated sea ice analysis. In order to reconcile the advantages of fully polarimetric acquisitions with the higher ground coverage of acquisitions with fewer polarimetric channels, hybrid polarimetric acquisitions offer a trade-off between the mentioned objectives. With the advent of the RISAT-1 satellite platform, we are able to explore the potential of hybrid dual pol acquisitions for sea ice analysis and classification. Our algorithmic approach for an automated sea ice classification consists of two steps. In the first step, we perform a feature extraction procedure. The resulting feature vectors are then ingested into a trained neural network classifier to arrive at a pixelwise supervised classification. We present first results on a dataset acquired off the eastern Greenland coast.
\end{abstract}

Index Terms - Sea Ice; Feature Extraction; SAR, Compact Pol; RISAT-1.

\section{INTRODUCTION}

Spaceborne SAR data has become a powerful means for sea ice monitoring since the launch of the first spaceborne platforms some decades ago (see eg. [1, 2, 3]). A common approach in automated sea ice classification based on SAR imagery is to first compute a vector of features pixelwise or for small neighborhoods of the image. While single-pol data can only be analyzed by classical image analysis for one channel (e.g. texture analysis), complex dual or fully polarimetric data allows the application of polarimetric analysis. After the

RISAT-1 data was kindly provided by KSAT, Norway. feature extraction, one then has to choose a classification approach. Our goal is to achieve a maximal degree of automation in our process chain, wherefore we adopt a supervised classification technique so we can include expert knowledge in our process chain. More precisely, a popular neural network library (FANN) is built into our algorithm. For operational purposes, the classifier needs to take into account variations due to region, season and in particular for different incidence angle ranges, when training according to expert information (in-situ observations, official ice charts): SAR images where ice classes are known with reasonable certainty serve as templates so the pertaining feature information from these images can be used train the classifier function to generate the reference output. Further details of such an neural network approach can be found in [1],[2], or [3]. For the details of the software tools, one may confer [4]. In our work we apply our neural-network based classification technique on RISAT-1 hybrid (CL-pol) mode imagery of sea ice. The RISAT platform is the first satellite borne SAR platform that is capable of acquiring hybrid dual polarimetric data (Right Circular Transmit Linear Receive: CL-Pol). Details for hybrid dual polarimetry can be found in [5] and the literature references therein.

\section{DATASET}

The dataset we used for our analysis were two adjacent and overlapping acquisitions by the RISAT- 1 satellite. Acquisition date was 2015/09/06, 18:13 (UTC), center locations of the two image frames are $78.14 \mathrm{~N}, 17.05 \mathrm{~W}$, and 77.89 N, $16.72 \mathrm{~W}$, off the Northeastern Greenland coast in the Atlantic. The image mode was Fine Resolution Stripmap (FRS1) with a resolution of 3 meters in azimuth and $2 \mathrm{~m}$ in range and a swath width of $25 \mathrm{~km}$. The transmitted C-band beam was right circular polarized $(\mathrm{R})$, the receiving linear polarization were vertical $(\mathrm{V})$ and horizontal $(\mathrm{H})$, a mode uniquely available in RISAT-1. Besides these circular-transmit/linearreceive modes (CL-pol), the FRS-1 stripmap mode, as well as the ScanSAR modes, can also be run in linear single-pol 
modes and dual-pol (HHHV, VVVH) mode. In a higher resolution of stripmap (FRS-2), also classical quad-pol images may be acquired. The dominant ice types we identified our dataset were open water/nilas (OW), young ice (YI), smooth first year ice (SFYI), and rough first year ice (RFYI), where the degree of roughness is to be understood as visually perceivable deformation.

\section{METHODOLOGY}

Our algorithmic approach consists of two steps: First we extract for each pixel a vector of polarimetric features. Then we feed each such vector into a neural network classifier. Lacking ground truth for the choice of training data, we relied on ancillary information from ice charts by the Danish meteorological service for the acquisition date and on expert visual judgement of the feature images and their RGB compositions. When the polarimetric parameters are derived from the covariance or coherency matrix, we used an $11 \times 11$ pixel sliding window for averaging purposes. The features/polarimetric parameters were extracted from the full resolution complex image layers $(R H, R V)$, with about $11300 \times 13900$ pixels per layer. The polarimetric parameters were taken from existing research in $[6,7,8,9,10]$, where [9] and [10] contains a survey of these features on simulated compact pol data with regard to sea ice classification. The basis of our analysis are the complex valued image layers $\left(S_{R H}, S_{R V}\right)$, where we define the notation

$$
S_{R H}=\sigma_{R H} \exp \left(j \varphi_{R H}\right) .
$$

Resulting features are the intensities

$$
\sigma_{R H}, \sigma_{R V}
$$

the phase difference

$$
\delta=\varphi_{R H}-\varphi_{R V},
$$

the circular (right) cross pol ratio

$$
\gamma=\frac{\left\langle\sigma_{R H}\right\rangle}{\left\langle\sigma_{R V}\right\rangle},
$$

conformity coefficient

$$
\mu=\frac{2 \operatorname{Im}\left\langle S_{R H} S_{R V}^{*}\right\rangle}{\left|S_{R H}\right|^{2}+\left|S_{R V}\right|^{2}} .
$$
by

Raneyet.al.[6] define the four Stokes parameters $S_{1}, S_{2}, S_{3}, S_{4}$

$$
\begin{gathered}
S_{1}=\left\langle\left|S_{R H}\right|^{2}+\left|S_{R V}\right|^{2}\right\rangle, \\
S_{2}=\left\langle\left|S_{R H}\right|^{2}-\left|S_{R V}\right|^{2}\right\rangle, \\
S_{3}=2 \operatorname{Re}\left\langle S_{R H} S_{R V}^{*}\right\rangle,
\end{gathered}
$$

and

$$
S_{4}=-2 \operatorname{Im}\left\langle S_{R H} S_{R V}^{*}\right\rangle .
$$

From these one derives the degree of polarization

$$
m=\sqrt{S_{2}^{2}+S_{3}^{2}+S_{4}^{2}} / S_{1}
$$

The degree of circularity is given by

$$
\sin 2 \chi=-S_{4} /\left(m S_{1}\right) .
$$

From $m$ and $\chi$ [6] derives the $m-\chi$ decomposition by

$$
\begin{gathered}
B=\left[m S_{1}(1-\sin 2 \chi) / 2\right]^{1 / 2}, \\
R=\left[m S_{1}(1+\sin 2 \chi) / 2\right]^{1 / 2}, \\
G=\left[S_{1}(1-m)\right]^{1 / 2},
\end{gathered}
$$

where B indicates the single-bounce (Bragg) backscatter, $R$ represents double-bounce, and $G$ corresponds to randomly polarized backscatter. The work of [7] furthermore propose the following features: circular (right) polarization ratio

$$
\mu_{c}=\frac{S_{1}-S_{4}}{S_{1}+S_{4}}
$$

ellipticity

$$
\mu_{E}=\frac{S_{4}}{S_{1}},
$$

relative phase

$$
\delta=\arctan \left(\frac{S_{4}}{S_{3}}\right)
$$

With $S_{1}, \delta$, and $m$, [7] derive the $m-\delta$ decomposition:

$$
\begin{gathered}
V_{R}=\sqrt{S_{1} m \frac{(1-\sin \delta)}{2},} \\
V_{G}=\sqrt{S_{1}(1-m),} \\
V_{B}=\sqrt{S_{1} m \frac{(1+\sin \delta)}{2}},
\end{gathered}
$$

where $V_{R}$ is related to double-bounce, $V_{G}$ is related to volumetric, and $V_{B}$ is related to surface scattering. [9] introduced the features correlation coefficient of amplitudes

$$
\rho=\frac{\sqrt{\left|\left\langle S_{R H} S_{R V}^{*}\right\rangle\right|}}{\sqrt{\left|S_{R H}\right|^{2}+\left|S_{R V}\right|^{2}}},
$$

When one defines the coherency matrix $T_{2}$ of the scattering vector $k=\left(S_{R H}, S_{R V}\right)^{t r}$, one can define (as is done in [9]) the Shannon entropy of intensity components

$$
H_{i}=2 \log \left(\frac{\pi e \operatorname{Tr}\left(T_{2}\right)}{2}\right),
$$



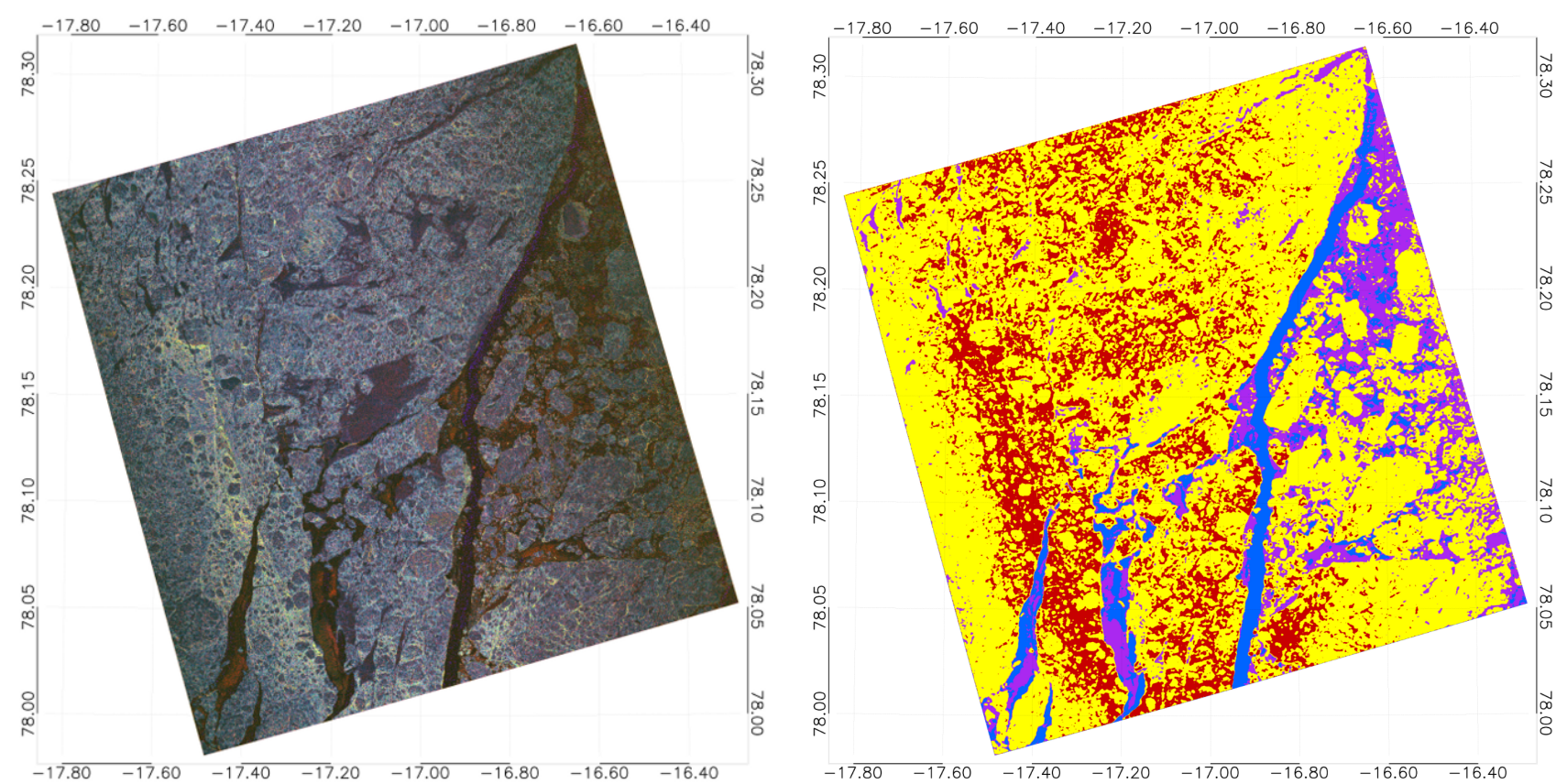

Fig. 1. Left: $m-\chi$ RGB composite image of RISAT-1 acquisition of 2015/09/06, 18:13:30 UTC. Right: Ice chart of RISAT-1 acquisition of 2015/09/06, top frame. Open water/nilas (OW) blue, young ice (IY) purple, smooth first year ice (SFYI) yellow, rough first year ice (RFYI) red.
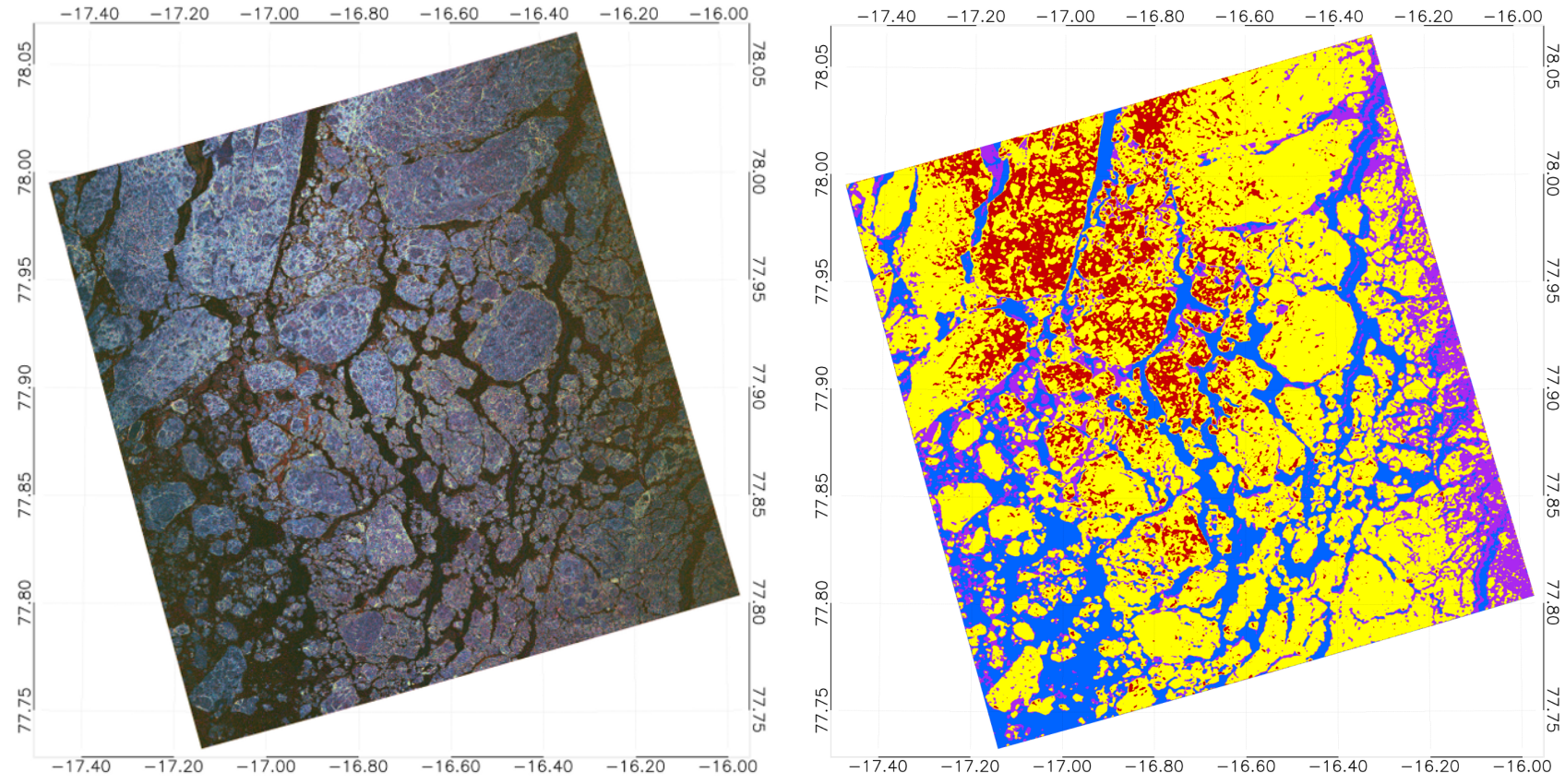

Fig. 2. Left: $m-\chi$ RGB composite image of RISAT-1 acquisition of 2015/09/06,18:13:26 UTC. Right: Ice chart of RISAT-1 acquisition of 2015/09/06,bottom frame. Open water/nilas (OW) blue, young ice (IY) purple, smooth first year ice (SFYI)yellow, rough first year ice (RFYI) red. 
and the Shannon entropy of the polarimetric component

$$
H_{p}=\log \left(\frac{4 \operatorname{det} T_{2}}{\operatorname{Tr}\left(T_{2}\right)^{2}}\right),
$$

where $\operatorname{Tr}(\cdot)$ denotes the matrix trace and det denotes the determinant.

For the implementation of the feature extraction we used IDL. The neural network was based on the FANN library, programmed in $\mathrm{C}$, which was built into our IDL implementation of the process chain. After feature extraction, the feature matrices were down-sampled by a user defined factor and rescaled to meet the requirements of the neural network input data range, resulting in an output classified image with dimension reduced by the user defined factor. The training data consisted of small patches of about 1000 pixels per ice type, taken only from the bottom frame. As mentioned these patches were determined by judgment of the polarimetric feature images in conjunction with DMI ice charts. In the network topology, we used two hidden layers with 14 and 16 hidden neurons, and for training of the neural network we used the RPROP algorithm. The algorithm was run on a virtual Linux machine with 6 logical cores (Intel Core i-7 3740 QM, 11GB RAM) with a total runtime of about 12-13 minutes which is in within the boundary of Near Real Time Requirement.

\section{RESULTS AND DISCUSSION}

The results of our classification can be seen in figures 1 and 2 , left hand image. Next to these images we display the m$\chi$ RGB composite of the acquisitions for comparison, see figures 1 and 2, righthand image. One can detect a strong match between the visual structures in these RGB images and the pertaining classified images. Open water/nilas areas are clearly detected throughout both acquisition frames. Also the young ice portions are classified noticably. Smooth first year ice portions, which appear as darker ice floes in the RGB image, are correctly detected, as well as the bright structures of more strongly deformed first year ice. With the help of mutual information analysis [2], we found out that contribution of $H_{i}, S_{1}, m-\chi_{B}$ and $H_{i}$ to classification stage is most significant.

\section{CONCLUSIONS}

We presented an algorithmic approach for automatically classifying ice types in hybrid dual-pol SAR imagery. We used a CL-pol dataset acquired through RISAT-1, the first satellite borne SAR system delivering genuine CL-pol products. Our process chain extracted polarimetric features and ingested the feature vectors in a neural network based classifier. Classification results show promising quality when juxtaposed to feature images. Future research will expand this approach to a wider array of CL-pol datasets, possibly with simultaneous in-situ data for validation, to explore the potential for operational purposes of sea ice classification.

\section{REFERENCES}

[1] A. V. Bogdanov, S. Sandven, O. M. Johannessen, V. Y. Alexandrov, and L. P. Bobylev, "Multisensor approach to automated classification of sea ice image data." IEEE T. Geoscience and Remote Sensing, vol. 43, no. 7, pp. 1648-1664, 2005.

[2] R. Ressel and S. Singha, "Comparing near coincident space borne $\mathrm{C}$ and $\mathrm{X}$ band fully polarimetric sar data for arctic sea ice classification," Remote Sensing, vol. 8, no. 3, p. 198, 2016. [Online]. Available: http://www.mdpi.com/2072-4292/8/3/198

[3] N. Y. Zakhvatkina, V. Alexandrov, O. M. Johannessen, S. Sandven, and I. Frolov, "Classification of sea ice types in ENVISAT synthetic aperture radar images," IEEE Transactions on Geoscience and Remote Sensing, vol. 51, pp. 2587-2600, 2013.

[4] S. Nissen, "Neural Networks made simple," Tech. Rep. 2, 2005.

[5] R. Shirvany, M. Chabert, and J.-Y. Tourneret, "Estimation of the Degree of Polarization for Hybrid/Compact and Linear Dual-Pol SAR Intensity Images: Principles and Applications," Geoscience and Remote Sensing, IEEE Transactions on, vol. 51, no. 1, pp. 539-551, Jan 2013.

[6] R. K. Raney, J. T. Cahill, G. Patterson, and D. B. J. Bussey, "The m-chi decomposition of hybrid dualpolarimetric radar data with application to lunar craters," Journal of Geophysical Research: Planets (19912012), vol. 117, no. E12, 2012.

[7] F. Charbonneau, B. Brisco, R. Raney, H. McNairn, C. Liu, P. Vachon, J. Shang, R. DeAbreu, C. Champagne, A. Merzouki et al., "Compact polarimetry overview and applications assessment," Canadian Journal of Remote Sensing, vol. 36, no. sup2, pp. S298S315, 2010.

[8] S. R. Cloude, D. G. Goodenough, and H. Chen, "Compact decomposition theory," Geoscience and Remote Sensing Letters, IEEE, vol. 9, no. 1, pp. 28-32, 2012.

[9] M. Dabboor and T. Geldsetzer, "Towards sea ice classification using simulated RADARSAT Constellation Mission compact polarimetric SAR imagery," Remote Sensing of Environment, vol. 140, pp. 189-195, 2014. [Online]. Available: http://www.sciencedirect. com/science/article/pii/S0034425713002939

[10] T. Geldsetzer, M. Arkett, T. Zagon, F. Charbonneau, J. J. Yackel, and R. K. Scharien, "All-season compactpolarimetry c-band sar observations of sea ice," Canadian Journal of Remote Sensing, vol. 41, no. 5, pp. 485504, 2015. 